\title{
Mujeres, sociedad civil y proceso de democratización en Corea del Sur
}

\author{
Women, civil society and the democratization \\ process in South Korea
}

\section{Bárbara Bavoleo y Paula Ladevito*}

\section{Resumen}

El artículo reflexiona acerca de la creciente participación de las mujeres surcoreanas en el espacio público a partir del decenio de 1980, expresada en la forma de movimientos, agrupaciones y asociaciones civiles. Se analiza la creciente presencia de estos y la significación de sus reivindicaciones dentro de la agenda política nacional, con el fin de dilucidar el grado de inclusión y participación alcanzado por este actor social en el modelo democrático de la República de Corea. Los movimientos de mujeres han ido cobrando cada vez mayor visibilidad y el estudio de los movimientos de mujeres y de su relación con la sociedad política permite dar cuenta de la intervención de la mujer en el espacio público, contribuyendo al conocimiento de su nueva condición en el escenario coreano actual, signado aún por la coexistencia de valores tradicionales y modernos. El trabajo vincula fuentes secundarias producto de las investigaciones de las autoras: «Sociedad civil y partidos políticos: Corea 1987-2002» e «Identidad y Género en Corea del Sur», de la primera y la segunda, respectivamente.

Palabras Clave: mujeres, participación, Corea del Sur, democratización, agenda política.

Becarias de CONICET < barbarabavoleo@yahoo.com.ar>; <paulaiadevito@yahoo.com.ar>. Recibido el 7 de abril de 2009; aceptado el 10 de julio de 2009. 


\section{Abstract}

The article reflects upon the increasing participation of South Korean women in the public scenario, as expressed in civil movements, groups and associations, since the 80 s. Their increasing presence and the significance of their demands in the context of the national political agenda is examined with the purpose of explaining the degree of inclusion and participation achieved by this social actor in the democratic model of the Republic of Korea. Women's movements have undoubtedly been acquiring increasing visibility and its study, together with its relationship with the political society sheds light on women's intervention in the public sphere, thus contributing to the understanding of their new status in the present Korean scenario, where traditional and modern values continue to coexist The paper links secondary sources product of the authors' research: : Civil Society and political parties: Korea 1987-2002» and «Identity and Gender in South Korea», respectively.

KEY WORDS: women, participation, South Korea, democratization, political agenda. 


\section{INTRODUCCIÓN}

A partir de la década de 1970, las mujeres coreanas comenzaron a participar en diferentes manifestaciones de la sociedad civil. Sin embargo, los temas de género, las reivindicaciones de sus derechos y la lucha por la igualdad de sexos quedaban de algún modo postergados en el marco de una sociedad aún fuertemente signada por la ideología confuciana y patriarcal. Cabe señalar que muchos de aquellos rasgos culturales sedimentados a lo largo de los distintos períodos que conformaron la sociedad Choson condicionarán el modo en que Corea se despliega en la modernidad y le otorgarán un sentido particular.

Resulta conveniente distinguir dos etapas evolutivas en el proceso de incorporación de las mujeres en el mundo público. Una primera etapa fue la que se extendió desde el decenio de 1970 hasta los primeros años de los años ochenta ${ }^{1}$, período durante el cual la participación de las mujeres fue limitada, marginal y focalizada en determinados niveles de la sociedad. Los temas femeninos encontraban espacio en el ámbito universitario $^{2}$, siendo las mujeres académicas quienes promovieron gradualmente el reconocimiento de los temas relaciona-

1 Cho, Young-sook (2007), The Role of Women's Organization for Gender Equality and Challenges Emerged, en Korea Women's Associations United Bulletin, p. 1.

2 En 1977, la Ewha Women's University establece el primer curso de estudios de la mujer. dos con la condición de la mujer como un problema social prioritario ${ }^{3}$.

En una segunda etapa -durante los primeros años de la década de 1980las activistas que participaban a titulo individual en varias organizaciones de la sociedad civil comenzaron a formar asociaciones, logrando que los temas de género obtuvieran una presencia cada vez mayor en la agenda de las organizaciones sociales. Sin embargo, solo en 1987 -año en que la República de Corea ${ }^{4}$ inició su transición formal hacia la democracia con la «Declaración de Reforma Democrática del 29 de junio» concretada por Roh Tae-woo- las mujeres alcanzaron mayor protagonismo en el escenario público, hecho que se enmarca en el proceso de reactivación de la sociedad civil en su conjunto. En diciembre de 1987, con la elección de Roh Tae-woo como Presidente, se inauguró la Sexta República, que constituyó un punto de inflexión en la historia política de Corea y que puso fin a las prolongadas décadas de gobiernos dictatoriales. Se trató de una elección limpia y libre que consagró la primera

3 Aunque estas primeras experiencias de participación femenina en la arena pública hayan quedado circunscriptas a determinados ámbitos, resulta pertinente señalar que, sin lugar a dudas, fue un momento fundacional con consecuencias no sólo para las mujeres sino para todos los integrantes del grupo familiar y la sociedad en general.

4 De aquí en adelante se utilizarán indistintamente los términos República de Corea y Corea para referirse a la parte sur de la península coreana. 
transferencia pacífica de mando. Así, durante su período de gobierno se adoptaron medidas de reforma liberal orientadas a resguardar los derechos políticos y las libertades civiles de los ciudadanos. Asimismo, se restableció el derecho de asociación ${ }^{5}$, hasta entonces vedado debido al efecto político de la confrontación ideológica entre el norte y el sur de la península y a la propia naturaleza del régimen surcoreano ${ }^{6}$.

En este contexto se asistió al resurgimiento de la sociedad civil, con un vigor comparable al alcanzado un poco más adelante, a mediados de la década de los años ochenta. Recordemos que ella había atravesado momentáneamente por un período de parálisis, como consecuencia de la materialización de algunas de sus demandas históricas, tales como la adopción del sistema de elección presidencial directa, la libertad de prensa y la autonomía de las instituciones académicas. Distintos grupos sociales: estudiantes, intelectuales, trabajadores, mujeres, campesinos y líderes religiosos reanudaron su intervención en la arena política. Sus

5 Diamond, Larry y Shin, Doh-chull (2000). Introduction: Institutional Reform and Democratic Consolidation in Korea, en Diamond, L. y Doh-chull Shin (eds.). Institutional Reform and Democratic Consolidation in Korea. California: Hoover Institution Press, p. 8.

6 Bavoleo, Bárbara (2007) Partidos políticos y sociedad civil en la democracia coreana. En Di Masi, J. y M. Crisconio (comp.) Corea y Argentina: percepciones mutuas desde una perspectiva regional', La Plata, Asociación Argentina de Estudios Coreanos, p. 446. demandas de reformas democráticas persistieron debido a que consideraban que la administración Roh era una continuación del régimen autoritario. En efecto, pese a las reformas liberales su gobierno aparecía como un autoritarismo liberalizado, razón por la cual a los ojos de la sociedad la lucha prodemocrática continuaba siendo vital ${ }^{7}$.

Los movimientos de mujeres -articulados a partir del reconocimiento de derechos económicos, políticos y sociales- alcanzaron cada vez mayor visibilidad y acompañaron este resurgimiento de la sociedad civil. Así fue que emergieron numerosas organizaciones $\mathrm{y}$ asociaciones de mujeres que dieron a conocer los problemas que enfrentaban. Algunos de sus reclamos lograron concretarse en leyes tales como la Ley de prevención de la violencia doméstica (1993) y la Ley básica de desarrollo de la mujer (1995), así como en la creación del sistema de cuotas de mediados de los años noventa, que dieron lugar a que aumentara la participación de la mujer en la política institucional.

Este artículo examina la creciente participación de las mujeres surcoreanas en el espacio público a partir de la década de 1980 y comenzará por dar a conocer las principales asociaciones de mujeres que acompañaron el proceso democratizador, para pasar luego a destacar las principales leyes relacionadas con la problemática de la mujer.

7 Kim, Sunhyuk (2002), Civil Society and Democratization. En C. Armstrong (Ed.). KoreanSociety. Civil Society, Democracy and the State. Londres,: Routledge, p. 98. 
Su identificación permitirá especular acerca del grado de inclusión alcanzado por este actor social en la democracia coreana. Se considera que el estudio de los movimientos de mujeres y de su intervención en el espacio público contribuye al conocimiento de la (re) configuración del sujeto femenino en el marco del proceso de modernización de la sociedad surcoreana.

\section{LA DEMOCRATIZACIÓN}

Y EL LUGAR DE LAS MUJERES

EN LA SOCIEDAD CIVIL

La transición política de un gobierno autoritario a una democracia electoral transformó tanto al movimiento por la democratización como a las agrupaciones de mujeres las que se propagaron considerablemente. Entre estas agrupaciones de mujeres cabe mencionar la Asociación de Mujeres para la Igualdad y la Paz, la Asociación de Mujeres para la Democracia y la Hermandad, y las agrupaciones Hot Line y Una Cultura Alternativa. Pero no solamente el factor político de cambio de régimen contribuyó a este crecimiento. Deben tenerse en cuenta también, entre otros, los siguientes factores: la participación de la mujer en el ámbito laboral y la experiencia directa de discriminación en el lugar de trabajo que aumentaron su conciencia de la presencia de problemas comunes;

8 En uno de sus artículos, Mera recapitula indicadores de la discriminación femenina la expansión del feminismo a nivel mundial y la presión internacional -de principios de los años ochenta- por alcanzar la igualdad de género, que llevó al gobierno coreano a crear el Instituto Coreano de Desarrollo de la Mujer y el Comité Nacional de Políticas de la Mujer, hecho que favoreció el estudio y el conocimiento de temas de mujeres tales como los sufrimientos y la lucha de las trabajadoras, que puso de manifiesto múltiples fuentes de opresión de la mujer. Sin duda, estas experiencias contribuyeron a la propagación de los movimientos femeninos?.

en relación con la actual situación de empleo, destacados por la Prof. Sun-ok Kim en una conferencia llevada a cabo en la Universidad de Belgrano de la Ciudad Autónoma de Buenos Aires. Entre las cifras mas representativas cabe mencionar las siguientes: en el sector de ventas y servicios el nivel de participación femenina es del $47 \%$ y su salario inferior en $50 \%$ al salario de los hombres; en cargos públicos y profesionales, las mujeres ocupan puestos de baja categoría no superando el $27 \%$ y, en lo que refiere a cargos políticos, la presencia femenina es prácticamente insignificante (de 109 diputados solo 3 son mujeres). La misma autora se refiere al problema de la segregación por género: es decir, las mujeres ingresan al mundo laboral insertándose en empleos de la economía informal en proporciones mucho más altas que los hombres-trabajadores. Véase Mera, Carolina (2004). Reflexiones acerca de los cambios en la mujer coreana: Corea y Argentina, en Mera, C. (comp.) Estudios Coreanos en América Latina. Buenos Aires-La Plata, Ediciones Al Margen, pp. 65-90.

$9 \quad$ Nam Jeong-lim (2000). Gender Politics in the Korean Transition to Democracy. Korean Studies. Vol. 24, pp. 96-97. 
El amplio desarrollo de estos movimientos se acompañó de un aumento de su cohesión, que se expresó en la formación de coaliciones. Así, en 1987, veintiuno de estos grupos voluntarios formaron la Asociación Unida de $\mathrm{Mu}$ jeres Coreanas (KWAU, por su sigla en inglés) con el objetivo de articular las reivindicaciones de otros grupos de mujeres (trabajadoras, campesinas, amas de casa urbanas, pobres urbanas). La KWAU difería de otras organizaciones de mujeres preexistentes o emergentes tanto por su declarada oposición al estado represivo como por su orientación feminista, que abogaba por la igualdad de género. Sus miembros fundadoras adoptaron la ideología y objetivos del movimiento Minjung ${ }^{10}$, tal como se desprende del primer volumen de su revista oficial, de acuerdo con el cual: "el objetivo de los movimientos de mujeres no debe restringirse a la obtención de derechos iguales a los hombres. Este debe orientarse a la transformación de las causas estructurales de la opresión de la mujer. Los movimientos de mujeres deben inscribirse en el movimiento más amplio de la democracia social que busca la igualdad de género, justicia y derechos humanos» ${ }^{11}$.

10 El movimiento Minjung (pueblo), surgido a principios de los años ochenta postulaba no solo la eliminación del régimen autoritario sino la evolución hacia un orden social alternativo, basándose en el análisis de las contradicciones del capitalismo y el cambio en la estructura de clases de la sociedad coreana.

11 Citado en Nam Jeong-lim, op. cit., p. 101.
En contraposición a la KWAU existían organizaciones de mujeres o asociaciones semi-voluntarias ${ }^{12}$-creadas por el régimen de Chun Doo-hwan, con el fin de colaborar con la represión estatal- categorizadas como Organizaciones de Masas Administradas ${ }^{13}$ (AMOs, por su sigla en inglés). Las AMOs fueron utilizadas como herramientas para la implementación de políticas de control de población y desarrollo económico y, para propagar las ideologías de seguridad nacional, anticomunismo y nacionalismo. Dentro de este tipo de organizaciones se encontraba el Consejo de Asociaciones de Mujeres Coreanas (KWAC, por su sigla en inglés) que había adoptado una postura conservadora y poco comprometida con la realidad político-social, y cuyas actividades se limitaban al trabajo caritativo, la protección de los derechos del consumidor, la promoción de la amistad entre sus miembros, y el ofrecimiento de clases y cursos para amas de casa y futuras esposas, denotando una falta de visión crítica y de conciencia feminista.

12 Las mismas se ubican en un punto intermedio entre la oposición de la KWAU y la sumisión de las AMOs ante la política del régimen. La mayoría de estas asociaciones intermedias eran de tipo religioso u ocupacional. Véase Moon, Seungsook (2002). Women and democratization in the Republic of Korea. The Good Society. Vol.11 No. 3, pp. 473-500.

13 Las AMOs se definen como "organizaciones civiles de masas creadas por los regímenes para implementar políticas públicas». Ver: Moon, Seungsook, op. cit. 
Durante el proceso de democratización se redujeron apreciablemente, las diferencias entre la KWAU y las AMO gracias al espacio de negociación inaugurado por el régimen democrático. Es decir, por un lado disminuyó el antagonismo entre la KWAU y el Estado debido a que su reivindicación central, la lucha contra la política estatal represiva, se diluyó en el contexto de la transición democrática y, por el otro, cesó el dominio estatal sobre las AMO, cuya participación política fue autorizada. En relación con la KWAU resulta importante señalar que hacia mediados de la década de 1990 comenzó a interesarse por los procesos electorales (tanto locales como nacionales) con vistas a influir en la toma de decisiones y en el diseño de las políticas públicas. Recién en 1998, con el gobierno de Kim Dae-jung, algunos de sus miembros fueron designados en cargos públicos, especialmente en organismos gubernamentales centrados en asuntos de la mujer. Al mismo tiempo, las AMO incorporaron la igualdad de género y la participación política de la mujer a sus temas de interés.

Estas asociaciones no estaban completamente desvinculadas de la actividad sindical. Ejemplo de ello fue el lazo de cooperación establecido entre la KWAU y la Asociación Unida de Mujeres Coreanas Trabajadoras (KWWAU, por su sigla en inglés). Esta última fue fundada en 1992 con la finalidad de agrupar a las distintas asociaciones regionales de mujeres trabajadoras, logrando de este modo centralizar y reforzar su poder. La KWWAU reclamaba fundamentalmente mejoras en las condiciones laborales e igualdad de posibilidades de acceso a puestos de trabajo ${ }^{14}$. Además, dichas asociaciones (la KWAU y las AMOs) mantenían contacto con el grupo de Mujeres Empresarias y Profesionales (BPW) y con la Asociación Coreana de Mujeres Universitarias (KAUW). Si bien las exigencias de estas organizaciones se circunscribían al ámbito de desarrollo de sus actividades, no pueden dejar de mencionarse debido a que han contribuido a la lucha por los derechos de la mujer. Otro grupo importante que es preciso mencionar es la Liga Coreana de Mujeres Votantes (KLWV, por su sigla en inglés) que, fundada en 1969 centró la atención en la participación y la educación de la mujer. Gracias a la liberalización política, la KLWV cobró mayor protagonismo y proyectó su preocupación a la difusión de los potenciales beneficios de la autonomía local hacia las mujeres votantes.

En Corea del Sur, los diversos movimientos de mujeres han contribuido al proceso de democratización en varios aspectos. Desde luego, ayudaron a ampliar la agenda política, incluyendo temas medioambientales y humanitarios. También, como en el caso de la KWAU, introdujeron temas esenciales para la creación de una sociedad democrática tales como la protección de la maternidad y los problemas de la niñez, las violaciones sexuales y el principio de igual

14 Para el año 1995, durante la presidencia de Kim Yong-sam, fue reconocida como agregado corporativo por el Ministerio de Trabajo de la República de Corea. 
remuneración por igual trabajo. Otro aporte fue la innovación en los modos de protesta y resistencia. Y por último, puede decirse que han contribuido a la transición democrática a través del reforzamiento del poder organizacional de los sindicatos: por ejemplo, la KWWAU adoptó la estrategia de expandir y revitalizar los comités de mujeres dentro de ellos.. Como sostiene Nam: "Al contrario de la creencia popular según la cual las mujeres coreanas son apolíticas y están excluidas de la politica nacional dominada por los hombres, muchas mujeres participaron en las luchas democráticas. Ellas tomaron parte de los movimientos democráticos a través de canales alternativos como las organizaciones de mujeres de base $y$ las protestas políticas a nivel local $\gg^{15}$.

\section{LAS REIVINDICACIONES FEMENINAS Y RESPUESTA LEGISLATIVA}

Las asociaciones de mujeres lograron compatibilizar sus intereses, haciendo converger sus esfuerzos para exigir acciones legislativas. Gracias a la formación de coaliciones entre diversos grupos de mujeres se logró la promulgación de algunas leyes. A manera de ejemplo, cabe mencionar la reforma de la Ley de Familia a fines de los años ochenta, la reforma del Decreto de Empleo Equitativo en 1989, 1995 y 2001,

15 Nam Jeong-lim (2000). Gender Politics in the Korean Transition to Democracy. Korean Studies. Vol. 24, pp.108-109. la aprobación y reforma del Decreto de Protección de la Niñez en 1990 y 2003, la promulgación de la Ley de Castigo a la Violencia Sexual y Protección de las Víctimas, en 1993, la aprobación de la Ley Especial de Castigo a la Violencia Doméstica en 1997, el establecimiento del sistema de cuotas para aumentar la participación de la mujer en el empleo público de mediados de los años noventa, la promulgación de la Ley de Prevención de la Prostitución y la Ley de Castigo a los Causantes de la Prostitución y Hechos Asociados en 2004. Todas ellas fueron resultado de la colaboración de la Asociación de Amas de Casa, la Federación de Clubes de Amas de Casa y la KWAU.

Sumada a esta convergencia de las asociaciones, el Estado abrió canales de negociación que generaron un cambio en su relación con el movimiento de mujeres. Por un lado, se manifestó como apoyo financiero del gobierno oficializado en 1995 con la promulgación de la Ley básica de desarrollo de la mujer ${ }^{16}$. Dicha Ley exigía la participación del gobierno central y local en el financiamiento de proyectos de organizaciones de mujeres destinados a la igualdad de género y el bienestar de la mujer. Por otro lado, estos canales de negociación permitieron que los movimientos de mujeres se involucraran en la reforma y la promulgación de nuevas

16 Antes de que se promulgara esta Ley, el Estado financiaba a las AMO y a las asociaciones semi-voluntarias a fin de ejercer control sobre ellas. Véase Monn, Seungsook, op. cit. 
leyes para la emancipación de la mujer. Entre las que lograron materializarse cabe mencionar: el Decreto de Cuidado Infantil, de 1991, el Decreto Especial de Violencia Sexual, de 1993 y su reforma de1997, y el Decreto de Prevención de la Violencia Doméstica de 1998.

La nueva relación entre las asociaciones de mujeres y el Estado incluyó el incremento y expansión de las agencias gubernamentales responsables de políticas públicas femeninas, tales como la implementación del sistema de inclusión de mujeres en puestos gerenciales de la administración pública, la expansión de la participación de estas en las comisiones internas del gobierno, la promoción del empleo de profesoras en las universidades públicas nacionales y el establecimiento de políticas presupuestarias diferenciadas por género ${ }^{17}$. Sin embargo, el hecho más destacado fue la creación del Ministerio de Igualdad de Género durante el gobierno de Kim Dae-jung que, a pesar de su limitado presupuesto, contribuyó significativamente a la expansión del discurso político femenino. Entre las principales actividades del Ministerio cabe citar las iniciativas para construir una perspectiva de género en el proceso de planeamiento presupuestario, la contribución a la reforma de la Ley de Maternidad, en 2001, que garantizó a las madres la licencia con goce de haberes y, más significativo aún, el compromiso de este organismo con la campaña que organizaciones civiles de mujeres lleva-

${ }^{17}$ Cho, Young-sook (2007). op. cit., pág. 4. ban adelante contra el sistema Hoju-je (sistema de registro familiar encabezado por los hombres) mediante el cual se amparaba la estructura patriarcal de la sociedad coreana. En 2005 se aprobó por mayoría absoluta en la legislatura la reforma de la Ley Civil para reemplazar al sistema Hoju-je; éxito que fue producto de 30 años de vigorosos esfuerzos de las mujeres coreanas ${ }^{18}$.

En las tareas de gestión orientadas a la obtención de derechos que resguardaran a las mujeres, la KWAU jugó un rol fundamental. Se encargó de difundir estos temas en los medios de comunicación social, de diseñar anteproyectos con la colaboración de profesionales especializados, de organizar reuniones de discusión con expertos y partidos políticos y utilizó las elecciones para ejercer presión sobre el gobierno y la legislatura para lograr la aprobación de las leyes. Como sostienen Madison y Jung: «... la KWAU permitió que las activistas feministas maximizaran las oportunidades politicas y se ganaran respeto como actores legítimos en el proceso formal de negociación ${ }^{19}$, no obstante, debido a la influencia que adquirió la KWAU, las organizaciones menores que no pertenecen a aquella tienden a verse excluidas del proceso de disposición de la agenda pública, de la distribución de recursos financieros

18 Madison, Sarah y Kyungja Jung (2007) Rethinking women's movements in changing contexts: Australia and South Korea, p. 16

19 Ibid., p.18. 
y de la participación en el proceso de toma de decisiones.

A lo largo del período de democratización puede advertirse un incremento del poder de presión de las asociaciones de mujeres para lograr que sus reivindicaciones adquirieran carácter legal. Los movimientos de mujeres no solo consiguieron participar en la esfera estatal con injerencia en la elaboración de normas, sino que conquistaron un espacio dentro de la más amplia sociedad civil coreana caracterizada por el predominio masculino. Así, tanto sus manifestaciones públicas como sus influencias en el ámbito institucional pueden leerse como indicadores, entre otros, del grado de inclusión conseguido por las mujeres dentro del modelo de democracia surcoreana.

\section{PARTICIPACIÓN POLÍTICA Y (RE) CONFIGURACIÓN DEL SUJETO FEMENINO}

La proliferación y paulatina consolidación de los movimientos de mujeres dentro de la sociedad civil forman parte del proceso de adaptación de Corea del Sur a la modernidad, dinamizado a partir de mediados del siglo XX. Como producto de las transformaciones a nivel económico, político, social y cultural ${ }^{20}$, las mujeres comenzaron

20 Corea del Sur atravesó por un proceso de profundos cambios que la convirtieron en una sociedad industrial, moderna y urbana. Su estructura agraria fue rápidamente reemplazada por una estructura productiva industrial que trajo aparejado un a traspasar los límites que las mantenían circunscritas al ámbito privado ${ }^{21}$. Así fue que ingresaron al mercado de trabajo, accedieron al sistema educativo y a la participación política. Esta progresiva inclusión de las mujeres en el espacio público se inscribe dentro del proceso de cambio del modelo de sociedad tradicional al modelo moderno. Los cambios en el sujeto femenino se relacionan con las variaciones en las estructuras sociales. Sin embargo, cabe señalar que: "... aunque desde los años sesenta muchas mujeres también empezaron a frecuentar las universidades y el nivel educativo de la mujer y su participación en la sociedad aumentó relevantemente, su consideración en todos los niveles todavía está muy mediatizada por su papel en el seno de la familia y su rol como madre» ${ }^{22}$.

La dicotomía entre lo público y lo privado vincula el primero con el Estado y el segundo con la familia y no solo refiere a una jerarquización de conceptos sino también a una sexualización de los mismos. Cada una de estas esferas de la sociedad se define a partir de un

cambio en el estilo de vida, los patrones y consumos culturales.

21 Dentro del modelo de sociedad tradicional, patriarcal, patrilocal y patrilineal, las mujeres coreanas estaban limitadas a su rol de esposas y madres. Véase Mera, Carolina. Op.Cit.

22 Doménech, Antonio José (2005). Mujer, Género y Familia en Corea, material publicado por la Cátedra Mera, Carrera de Ciencia Política, Facultad de Ciencias Sociales de la Universidad de Buenos Aires, p. 51. 
conjunto de atributos. A lo público se lo vincula con el Estado, lo objetivo, lo universal, lo abstracto, lo racional, lo literal y el trabajo productivo y, a lo privado, con la familia, lo subjetivo, lo particular, lo narrativo, lo emocional, la metáfora y el trabajo reproductivo. Estos atributos de público y privado refuerzan -en la modernidad- las jerarquías entre varones y mujeres, mientras que antes las mismas hallaban su respaldo en el orden de la naturaleza. Sobre este esquema binario continúan justificándose las desigualdades de género de la sociedad coreana actual. Un ejemplo claro de las desigualdades lo constituye la llamada «feminización de la pobreza» que se expresa en un $73 \%$ de mujeres subempleadas y en la Ley Básica de Familias Saludables, promulgada en 2003, que establece que las mujeres deben asumir la labor no remunerada de cuidar de los miembros de la familia. No obstante, en la Corea de hoy, a partir de fines de los años ochenta temas que en el contexto histórico tradicional fueron privados se han ido convirtiendo en asuntos públicos gracias al aumento de la participación política femenina.

El proceso de democratización abrió un espacio sociopolítico en el cual las asociaciones civiles pudieron emerger, expandir sus capacidades organizacionales y extender el rango de sus actividades a ámbitos públicos tales como temas medioambientales, garantías civiles, anticorrupción, derechos humanos y temas de género ${ }^{23}$. En

23 Kim Hyuk-rae (2004). Dilemmas in the este marco, el papel de las asociaciones de mujeres -que fue abriendo camino a la participación política femenina en el proceso de democratización- se constituyó en un elemento vital para las conquistas legislativas orientadas a resguardar los derechos de familia y de la mujer. Así, la mujer pudo trascender el ámbito privado e incursionar en el mundo de la política. Como sostiene Moon: "El pequeño cambio positivo en la representación de las mujeres en la política masculina requiere tanto del desarrollo de asociaciones de mujeres autónomas como del de un esquema alternativo de interpretación para entender el significado de las relaciones estado-sociedad» ${ }^{24}$. Si bien el tránsito por este camino ya se inició y las mujeres han logrado ganar posiciones en el ámbito civil, en los órganos legislativos y gubernamentales y en los partidos políticos, el mundo de la política en la sociedad surcoreana continúa siendo androcéntrico. Es decir, es un mundo regido por una ideología patriarcal -signada por los valores confucianosque ubica al hombre en el centro de la escena. Dicha impronta lejos de diluirse en el contexto de la modernidad se (re) significa en el marco del capitalismo.

Sin embargo, resulta innegable la proliferación de nuevas identidades signadas por la globalización y la diversidad cultural como consecuencia de las múltiples reestructuraciones de los

Making of Civil Society in Korean Political Reform. Journal of Contemporary Asia. Vol. 34. No. 1, p.3.

24 Moon, Seungsook. op. cit.,p. 41. 
escenarios locales e internacionales que caracterizan al mundo social actual ${ }^{25}$. Las transformaciones socioeconómicas y culturales encuentran como correlato una acentuación de los particularismos, es decir, una tendencia creciente a la diferenciación que se manifiesta en la emergencia de nuevas identidades políticas, multiculturales, étnicas, religiosas, sexuales, entre otras. Es decir, los «nuevos sujetos» del mundo globalizado definen sus formas de vida a partir de anclajes identitarios heterogéneos ${ }^{26}$. En este contexto, las retóricas y prácticas feministas tanto en Corea como en el resto del mundo, sin eludir las particularidades históricas de cada caso, han comenzado a cuestionar las categorías de sexo y género. El esfuerzo del pensamiento feminista es historizar el binarismo y repensar sus significados en los confines de la ciencia y la tecnología. Se trata de una búsqueda por comprender la relación entre el cuerpo físico y las influencias del discurso en el proceso de construcción del sexo-género.

\section{CONSIDERACIONES FINALES}

La democracia, entendida en términos amplios, requiere de la existencia

25 Véase: Sassen, S. (1999). La Ciudad Global. Buenos Aires: Eudeba (2003). Los espectros de la globalización. Buenos Aires: Fondo de Cultura Económica de Argentina.

26 Jameson, F. y S.Zizek (1998), Estudios Culturales: Reflexiones sobre el Multiculturalismo. Buenos Aires, Paidós. de equidad social y económica que habiliten una efectiva participación dentro de la escena política, tanto de las mujeres como de otras minorías sociales. En el caso de Corea del Sur, la precaria institucionalización partidaria de las décadas de los años ochenta y noventa y la consiguiente ausencia de canales regulares de transferencia de las demandas al Estado, convierte a los movimientos sociales en entidades que ejercen la representación aglutinando las demandas sociales ${ }^{27}$.

Como se ha mencionado, las organizaciones de mujeres fueron las encargadas de agrupar las demandas, no solo en relación con problemas de género sino también con solicitudes de inserción política. Como producto de su incorporación al espacio público se ubican las leyes que amparan la condición femenina. Es por eso que las mismas deben entenderse no como mera consecuencia de la instauración de un sistema de gobierno democrático sino como resultado de una lucha histórica que las mujeres, en tanto actor social, han emprendido en el contexto de la modernidad.

Una de las principales características de los movimientos de mujeres de finales de los años ochenta fue su preocupación ideológica por la transformación social que daba prioridad a los temas vinculados con la democracia

27 Bavoleo, Bárbara (2007), La institucionalización partidaria como elemento contingente. Revista Portes,. Tercera Época, Volumen 1, Número 1. Enero/Junio. Colima, México, pp. 39-52. 
y el nacionalismo respecto de los que enfatizaban su propia autonomía e independencia. Luego de lograda la democratización, sus actividades giraron hacia la realización de campañas relacionadas con aspectos de la vida diaria femenina. Así, las agrupaciones de mujeres comenzaron a tomar distancia del movimiento civil nacional estableciendo como contenido principal de su agenda las cuestiones de género e igualdad. La progresiva profundización democrática, hacia mediados del decenio de 1990 posibilitó el cambio de una relación de antagonismo con el Estado a una de negociación. Así, las asociaciones de mujeres participaron activamente en la formación de la agenda política y su influencia se destacó en la legislación nacional mediante la aprobación de importantes leyes como las que acá han sido destacadas. Durante los últimos dos gobiernos, Kim Dae-jung (1998-2002) y Roh Moohyun (2003-2007), se asistió a una alianza entre el Estado y el movimiento femenino que permitió que se nombrara ministros a cuatro mujeres, incluyendo a Han Myung-suk en 2006, en el cargo de Primer Ministro.

Por último, creemos que en el contexto de una sociedad capitalistapatriarcal, el reto que enfrentan los movimientos de mujeres es orientarse an principio hacia dos direcciones básicas. Por un lado, las mujeres deben defender tanto los derechos por la igualdad sexual (al trabajo, a la educación, a organizarse) como los derechos que avalen la especificidad de género (al aborto, a la anticoncepción, a la autodefensa, a los sindicatos, etc.). Pero si sus demandas se circunscriben a la lucha por la equidad y la igualdad de oportunidades o a la reivindicación de aquellas cuestiones que definen la naturaleza femenina, existe el riesgo caer en esencialismos. En el primer caso, negando la sexualización, pero no la jerarquización que establece el par dicotómico público-privado y, en el segundo, revirtiendo la jerarquízación pero no la sexualización. Es por eso que el pensamiento feminista deberá plantearse sistemas complejos que permitan explicar los cambios y las realidades actuales, teniendo en consideración otros sujetos, otras miradas y saberes diversos que modifican las instituciones en el día a día ${ }^{28}$. Por otro lado, es indispensablel que los movimientos de mujeres se diversifiquen, amplíen sus bases y se consoliden para acceder a la instancia de negociación con el Estado

28 Dentro del campo de producciones teóricas del feminismo occidental, en las últimas décadas se ha venido desarrollando la llamada tercera vía o posición, cuyas reflexiones en torno a la cuestión de la identidad y el género cuestionan algunas de las premisas básicas del feminismo cultural y del feminismo post-estructuralista. Los aportes de Judith Butler (2001) y Teresa De Laurentis forman parte de esta renovada línea de pensamiento. El debate al interior del feminismo y las contribuciones de las autoras pueden consultarse en: Acoff, Linda (2001) Feminismo cultural versus posestructuralismo: la crisis de la identidad en la teoría feminista, en Navarro, M. y C. Stimpson, (comp.). Nuevas Direcciones. Buenos Aires: Fondo de Cultura Económica de Argentina, pp. 65-106. 
demostrando una capacidad de respuesta relativamente autónoma.

Es innegable que los movimientos de mujeres coreanas adquirieron relevancia en el contexto sociopolítico contemporáneo y que lograron avances significativos en materia legislativa. Sin embargo, este es solo uno de los aspectos del proceso de modernización del modelo de mujer. Otras aproximaciones podrán enriquecer este enfoque indagando los espacios de sociabilidad y la experiencia de las mujeres coreanas, igualmente configuradores de su identidad.

\section{BIBLIOGRAFÍA}

Acoff, Linda (2001). Feminismo cultural versus posestructuralismo: la crisis de la identidad en la teoría feminista. En Navarro, M. y C. Stimpson (comps.). Nuevas Direcciones, Buenos Aires: Fondo de Cultura Económica de Argentina. pp. 65-106.

Bavoleo, Bárbara (2005), Sociedad Civil en el Proceso de Consolidación Democrática. República de Corea: 1995-2000, publicación electrónica y en CD del II Encuentro Latinoamericano de Estudios Coreanos, El Colegio de México - Korea Foundation.

Bavoleo, Bárbara (2007), Partidos políticos y sociedad civil en la democracia coreana. En Di Masi, J. y M. Crisconio (comps.), Corea y Argentina: percepciones mutuas desde una perspectiva regional, La Plata: Asociación Argentina de Estudios Coreanos, pp. 445-458.

Bavoleo, Bárbara (2007), La institucionalización partidaria como elemento contingente, Revista Portes. Tercera Época, Volumen 1, Número 1. Enero/Junio, Colima, México, pp. 39-52.

Butler, Judith (2001). El género en disputa. El feminismo y la subversión de identidad,. México DF: Paidós.
Cho, Hein (1997). The Historical Origin of Civil Society in Korea. Korea Journal. Summer. pp. 24-41.

Cho, Young-sook (2007), The Role of Women's Organization for Gender Equality and Challenges Emerged, Korea Women's Associations United Bulletin, pp.. 1-10.

Choi, Jang-jip (2000), Democratization, Civil Society, and the Civil Social Movement in Korea: The Significance of the Citizens' Alliance for the 2000 General Elections, Korea Journal, Autumn, pp. 26-57.

De Lauretis, Teresa (1996), La tecnología del género. Revista Mora No 2,pp.6-34.

Diamond, Larry y Shin, Doh-chull (2000). Introduction: Institutional Reform and Democratic Consolidation in Korea. En Diamond, L. y Doh-chull Shin (eds.). Institutional Reform and Democratic Consolidation in Korea, California: Hoover Institution Press,pp.1-42.

Doménech, Antonio José (2005). Mujer, Género y Familia en Corea, material publicado por la Cátedra Mera, Carrera de Ciencia Política, Facultad de Ciencias Sociales de la Universidad de Buenos Aires.

Han, Sang-jin (1997). The Public Sphere and Democracy in Korea. A Debate on Civil Society, Korea Journal, Winter, pp.78-97. Iadevito, Paula Marina (2005), Corea tradicional y moderna: espacios de construcción de la identidad femenina. En E. Oviedo (comp.) Corea... una mirada desde Argentina, Rosario: Editorial de la Universidad Nacional de Rosario, pp. 271-290.

Iadevito, Paula y Laura Zambrini (2007), Identidad, Género y Subjetividad en el mundo actual: aportes del feminismo y la filosofía, publicación en CD de las Jornadas Pre-Alas Preparatorias del XXVI Congreso ALAS Guadalajara 2007-Buenos Aires-Argentina, Facultad de Ciencias Sociales - Universidad de Buenos Aires.

Jameson, F. y S.Zizek (1998). Estudios Culturales: Reflexiones sobre el Multiculturalism, Buenos Aires: Paidós.

Kim Hyuk-rae (2004). Dilemmas in the Making of Civil Society in Korean Political 
Reform, Journal of Contemporary Asia, Vol. 34 - No. 1, pp.3-23.

Kim, Hyuk-rae (2002). Unraveling Civil Society in South Korea: Old Discourses and New Visions, Korea Observer, Vol.33 - No. 4, Winter, pp. 541-568.

Kim, Sunhyuk (1997), State and Civil Society in South Korea's Democratic Consolidation: Is the Battle Really over?, Asian Survey, Vol. 37 - No. 12. Dec. pp.1135-1144.

Kim, Sunhyuk (2002), Civil Society and Democratization. En C. Armstrong (ed.). Korean Society. Civil Society, Democracy and the State. Londres, Routledge,pp. 92-108.

Kim, Sunhyuk (2003), Civil Society in Democratizing Korea. En Kim, Samuel (ed.), Korea's democratization, Cambridge, Cambridge University Press, pp. 81-106.

Lief Palley, Marian (1990), Women's Status in South Korea: Tradition and Change, Asian Survey, Vol. 30 - No.12, pp.1136-1153.

Lim, Hy-sop (2000), Historical Development of Civil Social Movements in Korea: Trajectories and Issues. Korea Journal. Autumn, pp. 5-25.

Madison, Sarah y Kyungja Jung (2007), Rethinking women's movements in changing contexts: Australia and South Korea. Disponible en: http://ipsa-rc19.anu.edu.au/Maddison \%20Jung.ipsa06\%20.pdf, pp.1-25.
Mera, Carolina (2004), Reflexiones acerca de los cambios en la mujer coreana: Corea y Argentina. En C. Mera (comp.). Estudios Coreanos en América Latina. Buenos Aires-La Plata, Ediciones Al Margen, pp.65-90.

Moon, Seungsook (2002), Women and democratization in the Republic of Korea, The Good Society, Vol.11 - No. 3, pp.36-42.

Nam, Jeong-lim (2000), Gender Politics in the Korean transition to democracy. Korean Studies, Vol. 24, pp.94-112.

Pak, Sejin (1998), Two Forces of Democratization in Korea. Journal of Contemporary Asia , 28 - No.1,pp. 45-73.

Sassen, S. (1999), La Ciudad Global. Buenos Aires, Eudeba.

Sassen, S. (2003), Los espectros de la globalización, Buenos Aires: Fondo de Cultura Económica de Argentina.

Seong, Kyung-ryung (2000), Civil Society and Democratic Consolidation in South Korea: Great Achievements and Remaining Problems. En Diamond, L. y Kim, Byung-kook, Consolidating democracy in South Korea, colaborado, Lynne Rienner Publishers,pp.87-110. 